Historic, Archive Document

Do not assume content reflects current scientific knowledge, policies, or practices. 


\title{
Bulletin No. 1
}

September 25, 1931

\section{Thomas B. Meehan Co.}

\author{
DRESHER, PA.
}

Telegraph address, Ambler, Pa.

READ TERMS

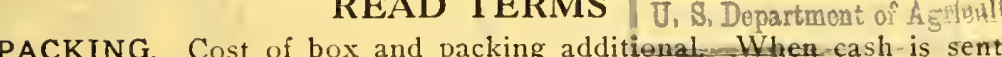
with order add 15 per cent. of the bill of goods to cover packing charges. DELIVERY. To freight and express station, Dresher, Pa.

QUANTITIES. 5 of any one variety and size, furnished at the rate quoted per variety and size add five cents each to the rate per ten. On trees add ten cents to the rate per ten.

RESPONSIBILITY. After goods are placed on board cars here, we assune no responsibility for their sale delivery or any injury caused by

ACANTHOPANAX

175 pentaphylla, $1 \frac{1 / 2}{2}$ to 2
300 pentaphyylla, 2 to 3 ft.
300 pentaphylla, 3 to 4 ft
100

Per 10 Per 100

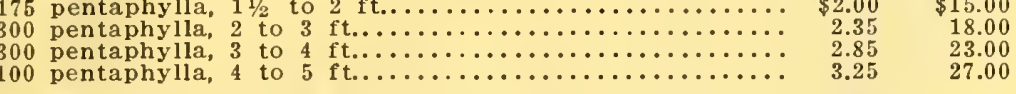

ACER-IIaple

36 dasycarpum, Silver, 6 to 8
212 dasycarpum, Silver, 8 to 10

$212 \mathrm{~d}$

1 dasycarpum,

38 dasycar

dasycarpum, Silver, $23 / 2$ to 3 in. cal.....

5 dasycarpum Wierii, cut-leaved, 6 to 8 ft.

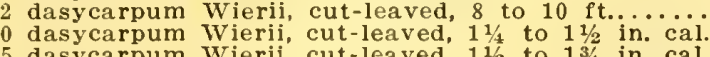

5 dasycarpum Wierii, cut-leaved, 11 to 13 is in. cal.

5 ginnale, $1 \frac{1 / 2}{2}$

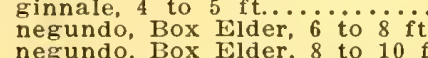

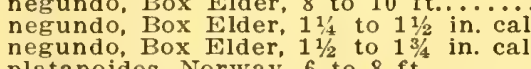

(6) platanois

platanoides, Norway, $11 / 4$ to $11 / 2$ in cal.:

01 platanoides

platanoide

platanoides, Norway, $2 \%$ to 3 in. cal...

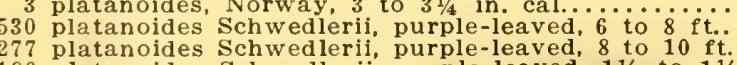

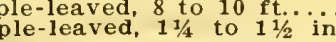

45 platanoides schivedierii, purple-leaved, $1 \frac{1 \% 2}{2} 1 \frac{3}{4}$ in

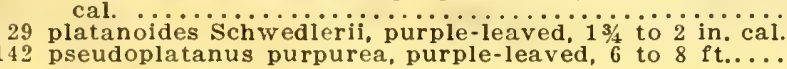

94 polymorphum atropurpureum, blood-leaved, 12 to 15

86 polymorphum atropurpureum, blood-leaved, 15 to is $\$ 1$.

32 polymorphum atropurpureum, blood-1eaved, $1 \%$ to 2

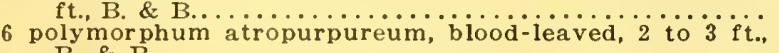

ALTHAEA-IIIBSCUS-Rose of Sharon

150 anemonaeflora, double pink, $1 \frac{1 / 2}{2}$ to $2 \mathrm{ft}$

anemonaeflora, double pink, 3 to $4 \mathrm{ft}$.

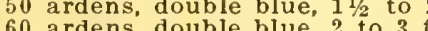

5 ardens, double blue,

100 Boule de Feu, double re

政,

Ded 2 to 3

50 Duc de Brabant, double red, 4 to 5

50 Jean de Arc, double white white.

purpurea semi-plena, double purple, 1 i 1 , to $2 . .$.

purpurea semi-plena, double purple, 2 to $33 \mathrm{ft}$.

rubis, single red, $11 / 2$ to 2

24 rubis, single red, 3 to

415 Snowdrift, single white, $11 / 2$ to 2 .

Snowdrift, single white, 3 to 4

0 totus albus, single white,

00 variegatas, varng

530 variegata, variegated-leaved, $11 \frac{1}{2}$ to 2 ft

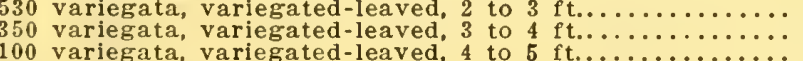
LyoND-See P'runus

M MELANCHIER-Shad Bush

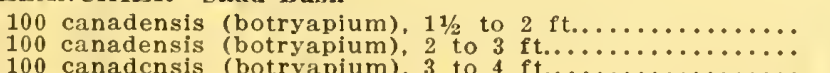
AMORPHA-Indige Shrub

170 fruticosa, 2 to 3
150 fruticosa, 3 to 4

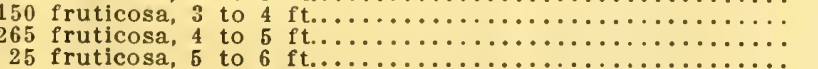
AMPELOPSIS

100 heterophylla, cut-leaved, 2 year.

200 quinquefolia, Virginia Creeper, 1 year

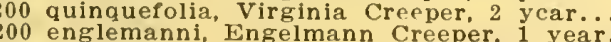

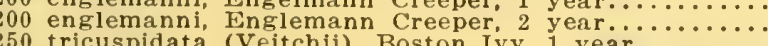

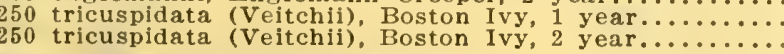

A 1 rgdalus NANA-Flowerlng Peaeh
200 Pink, Red and White, 3 to $4 \mathrm{ft}$.

$\begin{array}{rr}4.50 & 35.00 \\ 6.50 & 50.00 \\ 9.00 & 75.00\end{array}$

15.00
150.00

200.00

250.00
300.00

65.00
85.00

175.00
25.00

30.00
50.00

35.00
50.00
85.00

100.00

200.00
275.00

375.00
450.00

525.00
600.00

${ }_{225.00}^{1500}$

325.00

400.00
475.00

125.00

Per 10

20.00

25.00

35.00

Per 10 Per 100

$\$ 1.50 \quad \$ 10.00$

$\begin{array}{ll}1.50 & 10.00 \\ 2.00 & 15.00\end{array}$

15.00
20.00
25.00

BALIA-lle reules Club

20 spinosa, $1 \frac{11 / 2}{\text { to }} 2$

5 spinosa, 4 to $45 \mathrm{f}$ 
ARoNIA-Pyrus-Chokeberry

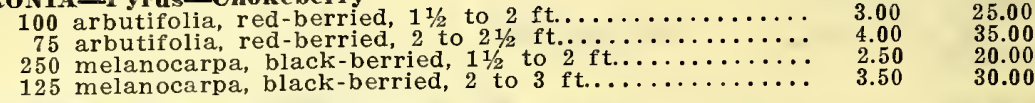

BENZOIN-(Lindera)-Spiee Rush

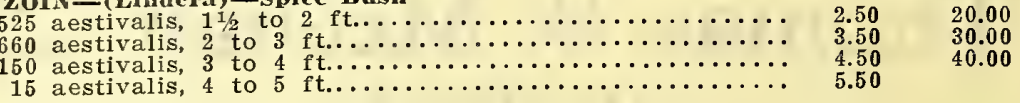

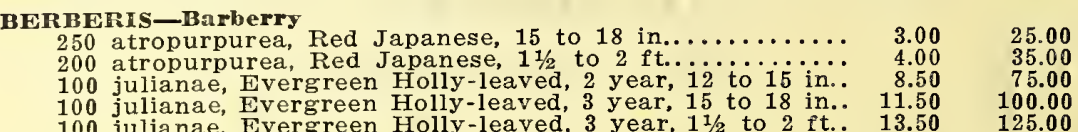

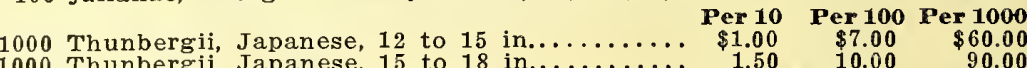

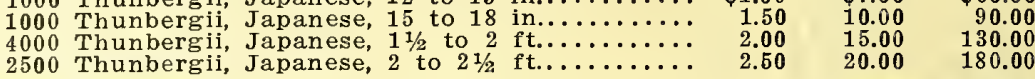

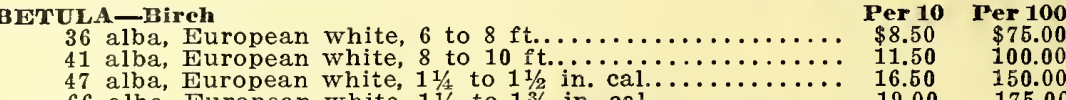

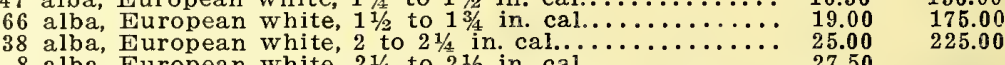

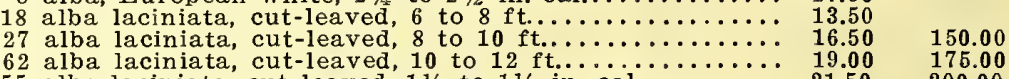

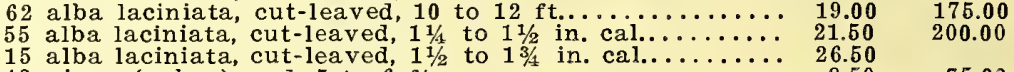

88 nigra (rubra), red, 5 to 6

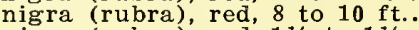

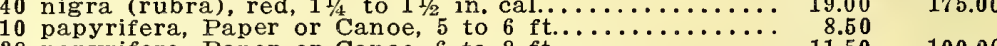

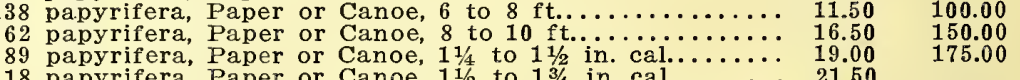

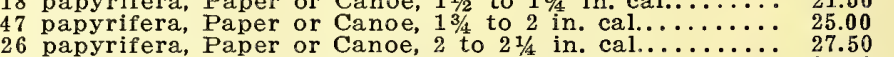

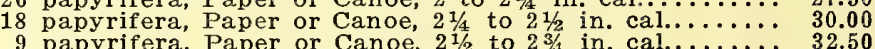

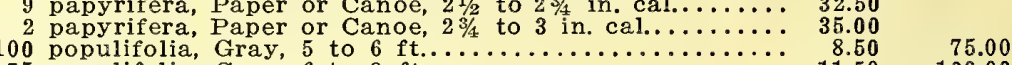

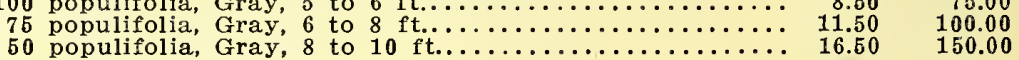

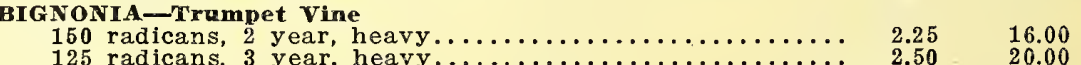

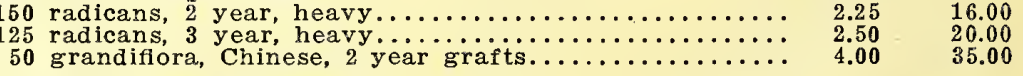

\section{BUCKTHORN-See Rhamnus}

BUDDLEIA-Butterfly Bush

CALLICARPA-ChInese Beauty Prut

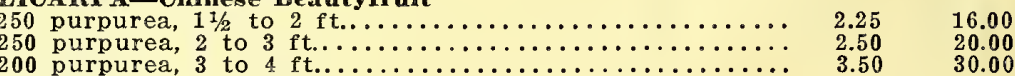

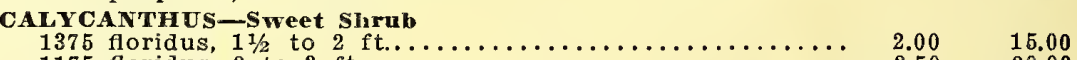

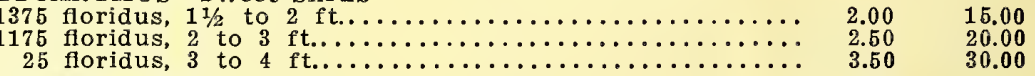

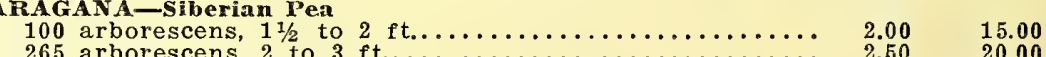

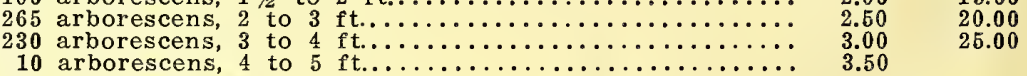

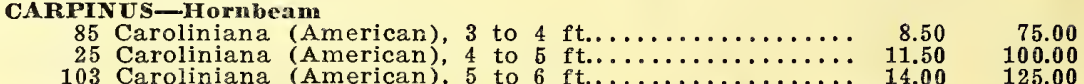

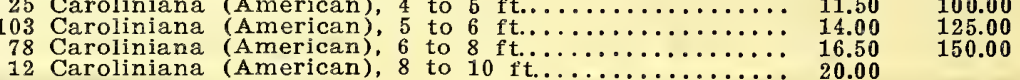

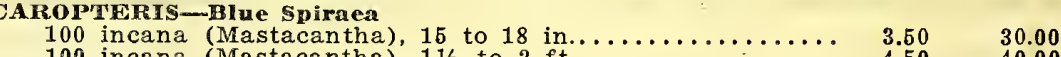
100 incana (Mastacantha), $11 / 2$ to 2 ft......................... $4.50 \quad 40.00$

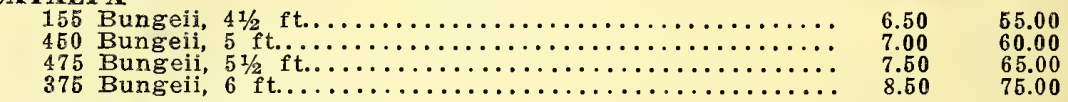

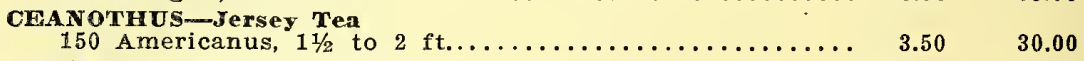

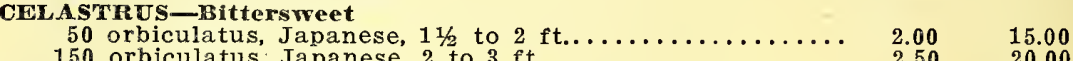

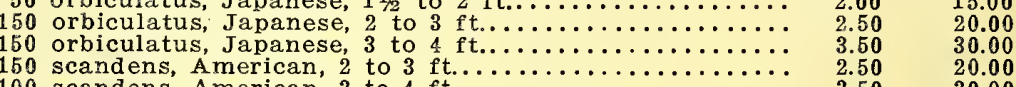
100 scandens, American, 3 to 4 ft..................... $3.50 \quad 30.00$

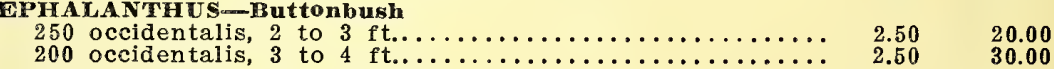

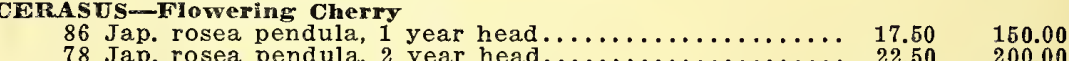

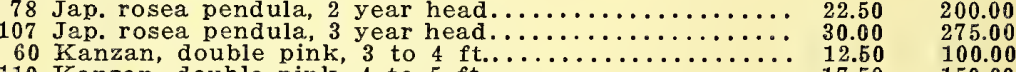

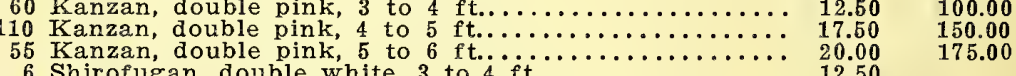

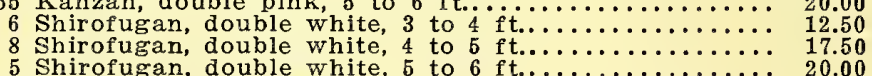
CIDIPHYLLUM-Katsura Tree

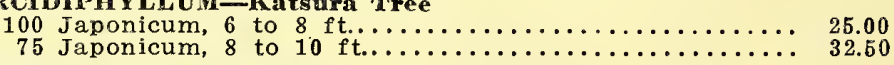

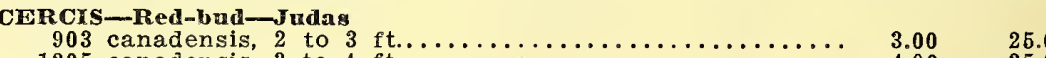

591
590
150 canadensisis,

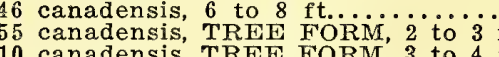

110 canadensis, TREE FORM, 3 to 4 ft
60 canadensis, TREE FORM, 4 to 5 f

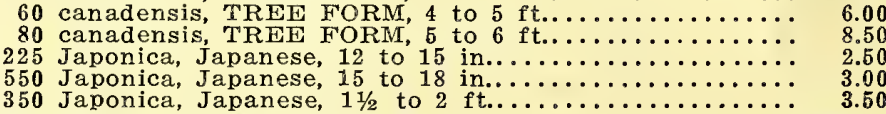

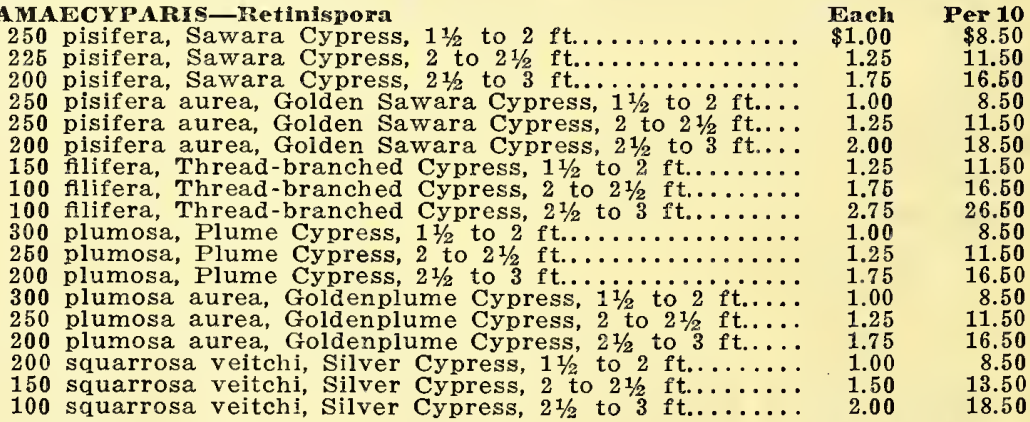

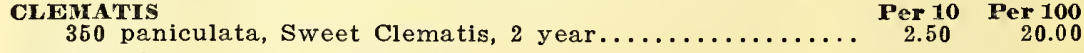

CLETHRA-Sweet Pepper Bush

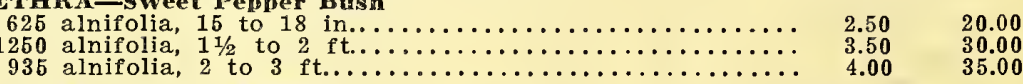

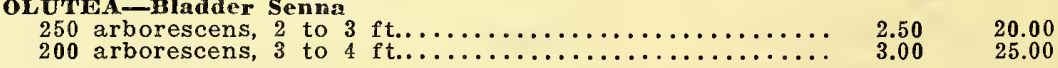

CORNDS-Dogwood

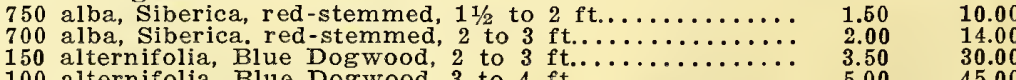

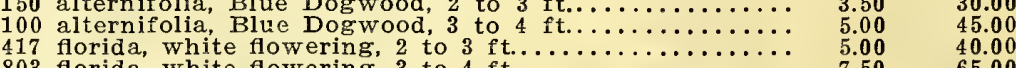

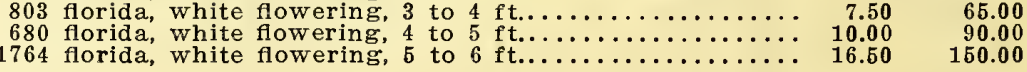


THOMAS B. MEEHAN CO., DRESHER, PA.-BULLETIN No. 1

ConNus-Dogwood-(Continued)

445 florida, white flowering, 6 to 8 ft..
291 florida rubra, Pink Dogwood, 2 to

847 florida rubra Pink Dogwood, 4 to 4 .

1035 florida rubra, Pink Dogwood, 4 to 5 th 6 r

150 kousa, Kousa Dogwood, 3 to 4 ft.

100 Mrascula, Cornelian Cherry, 2 to 3 ft.

400 paniculata (canadensis),

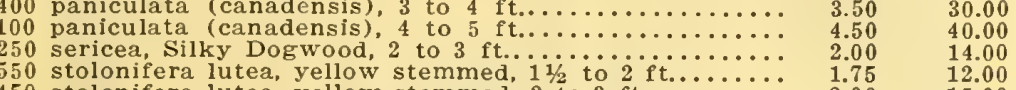

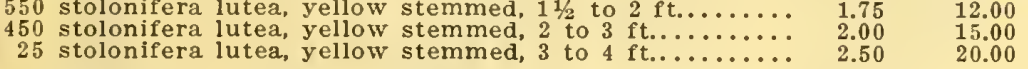

CORYLUS-Fibert

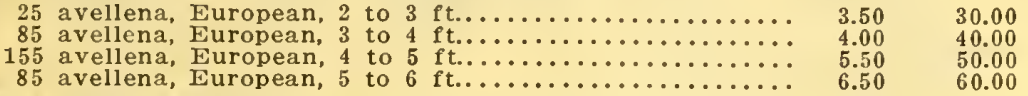

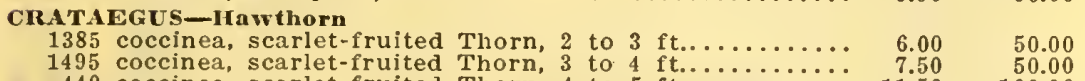

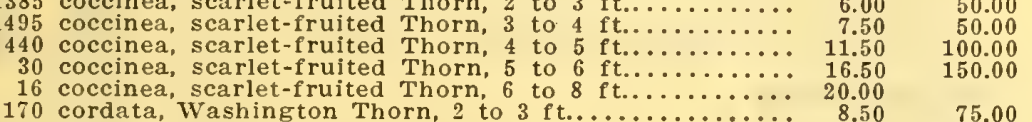

170 cordata, Washington Thorn,
215 cordata, Washington Thorn,

1065 crus galli, Cockspur Thorn,

255 crus galli, Cockspur Thorn,

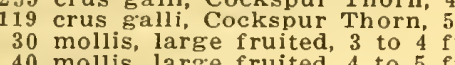

200 oxycantha, English, 2 to 3

105 oxycantha, English, 3 to 4

65 oxycantha, English,

50 oxycantha, English,
23 Paul's double scarle

152 Paul's double scarlet, 3 to 4 t

45 Paul's double scarlet, 6 to 8 ft............................. 12.50

16 pyracantha, fire thorn, 2 to $21 \frac{1}{2}$ \&t., B. \& B........... $\begin{gathered}\text { Each } \\ \$ 2.25\end{gathered}$

CYDONIA-Japanese Quinee-Fire Bush

75.00
100.00
125.00
60.00

60.00
85.00
115.00
135.00

135.00
85.00
115.00
3000

40.00
60.00
90.00

90.00
150.00
50.00
75.00

3800 Japonicum, 11 to $1 \frac{1 / 2}{2} \mathrm{ft}$.

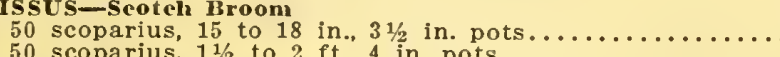

Per 10 19.00

$P$ Per 100
$\$ 12.00$
15.00

PHNE-Rose Daphne

100 cneorum, 6 to 9 in..
75 cneorum, 9 to 12 in.
75 cneorum, 12 to 15 in.

DESMODIUN-Bush Clover

3.50

30.00
35.00

Per 10
$\$ 6.50$
10.00

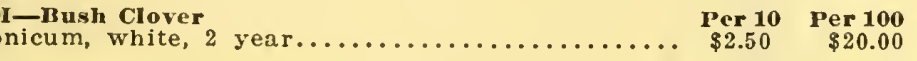

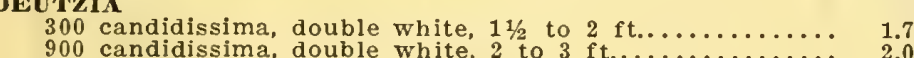

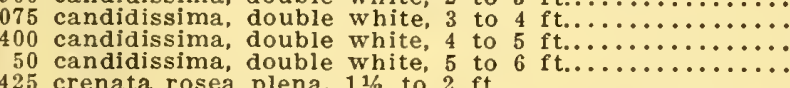

25 crenata rosea plena, 1

155 crenata rosea plena, 3 to 3 ft.

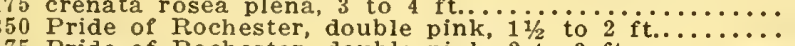

Pride of Rochester, double pink, 2 to $3 \mathrm{ft}$

250 Pride of Rochester, double pink, 4 to $5 \mathrm{f}$
325 gracilis, dwarf, single white, 15 to 18 in.
625 gracilis, dwarr, single white, $11 / 2$ to $2 \mathrm{ft}$...
1075

0
gracilis rosea, single pink, 15 to 18 in.
gracilis rosea, single pink, $1 / 2$ to $2 \mathrm{ft}$.

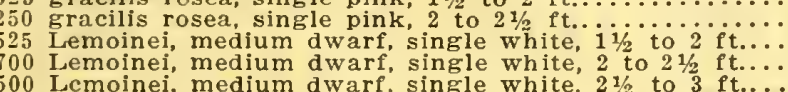

DERVILLA-Dwarf Bush Honeysuekle

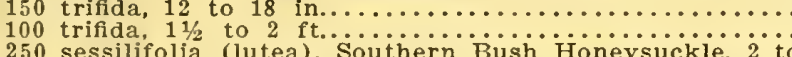

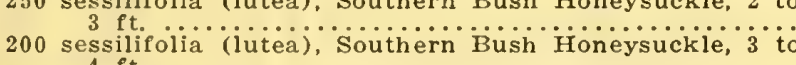

$\begin{array}{ll}1.75 & 12.00 \\ 2.00 & 14.00 \\ 2.35 & 18.00 \\ 2.75 & 22.00 \\ 3.50 & 30.00 \\ 1.75 & 12.00 \\ 2.00 & 14.00 \\ 2.35 & 18.00 \\ 1.75 & 12.00 \\ 2.00 & 14.00 \\ 2.35 & 18.00 \\ 2.75 & 22.00 \\ 2.00 & 14.00 \\ 2.35 & 18.00 \\ 2.85 & 23.00 \\ 2.35 & 18.00 \\ 2.85 & 23.00 \\ 3.50 & 30.00 \\ 2.00 & 15.00 \\ 2.50 & 20.00 \\ 3.00 & 25.00\end{array}$

$\begin{array}{ll}3.00 & 25.00 \\ 4.00 & 35.00\end{array}$

25.00

30.00

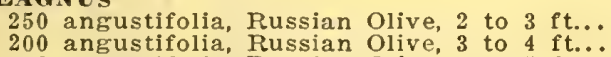

150 angustifolia, Russian Olive, 4 to $5 \mathrm{ft}$.

150 longipes, Cherry Eleagnus, 3 .

50 umbellata, Autumn Eleagnus,

150 umbellata, Autumn Elea

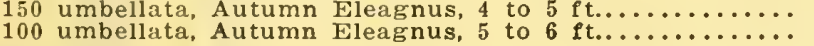

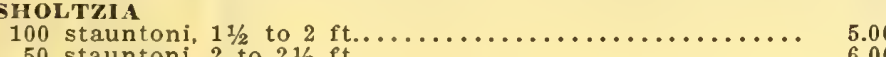

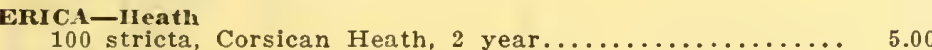

EUONYMUS

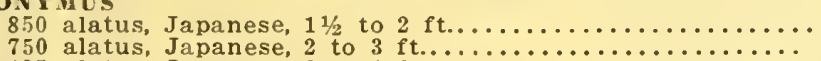

485 alatus, Japanese, 3 to 4 t

110 alatus, Japanese, 4 to $5 \mathrm{ft}$.

150 Americana, Brook Euonymus, 2 to 3 ft.

100 bungeanus, Winterberry Euonymus, 3 to 4 tit.

150 Europeaus, European Burningbush, 2 to $3 \mathrm{ft}$. Euroush

100 Europeaus,

425 radicans, creeping, 6 to 9 in.............. 9 to 12 in.

40 radicans variegata,

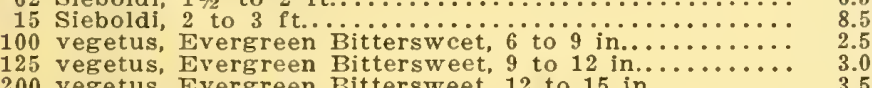

XOCHORDA-Pearl Rush

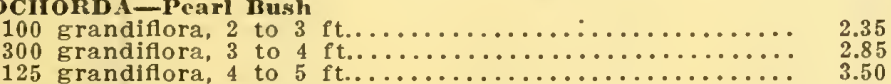

125 rrand

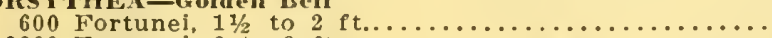

3100 Fortunei, 2 to 3 ft.

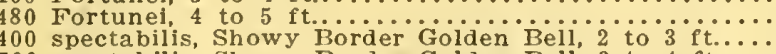

500 spectabilis, Showy Border Golden Bell, 3 to $4 \mathrm{ft}$.

115 suspensa, $1 \frac{112}{2}$ to $2 \mathrm{ft}$.

25 suspensa, 3 to $4 \mathrm{ft}$.......

400 viridissima

700 viridissim

FRAXINUS-A

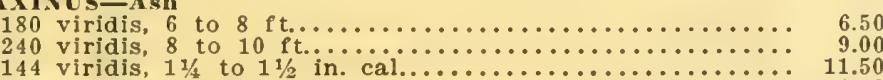

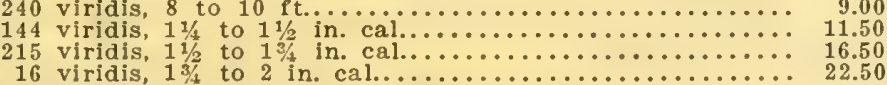

GLEDITSCHIA-Honey Locust

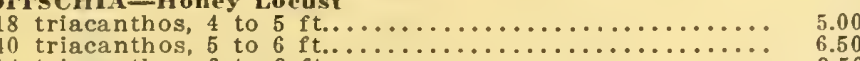

14 triacanthos, 6 to 8 ft................................

16.00
20.00

30.00
35.00
50.00
35.00

50.00
70.00
85.00

40.00
50.00

45.00

20.00
30.00

30.00
40.00
75.00

90.00
25.00

25.00
35.00
30.00

40.00
20.00

40.00
12.00

12.00
50.00

20.00
25.00
30.00

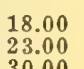

$\begin{array}{ll}1.75 & 12.00 \\ 2.00 & 14.00\end{array}$

11.00
18.00

25.00
16.00

20.00
30.00
16.00

20.00
25.00
12.00

14.00
18.00
25.00

50.00
75.00

100.00
150.00

40.00
50.00

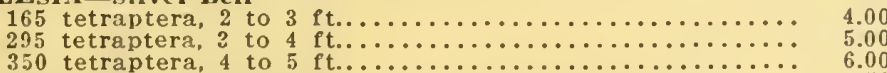

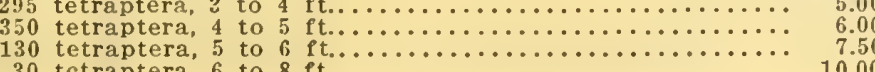

30.00
40.00
50.00
65.00
85.00 


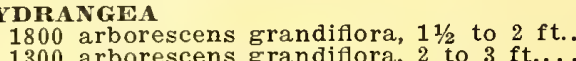

150 paniculata, type, $11 / 2$ to $2 \mathrm{ft}$
250 paniculata, type, 2 to $3 \mathrm{ft}$...
400 paniculata

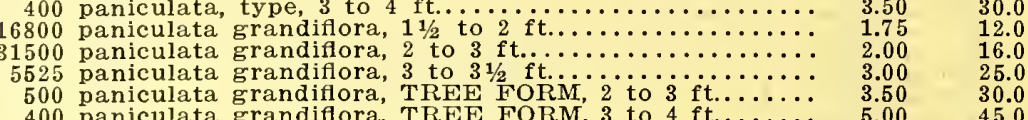

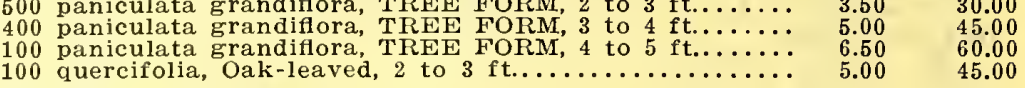

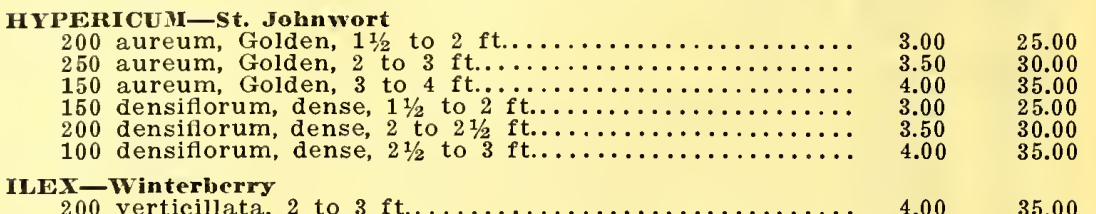

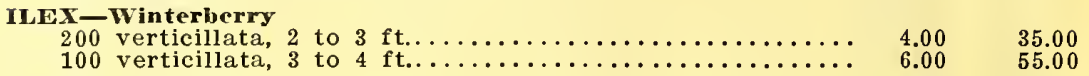

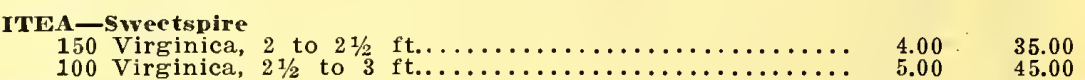

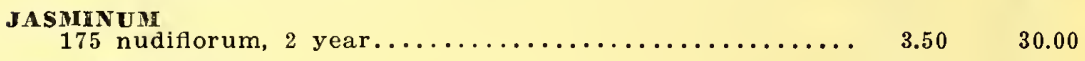

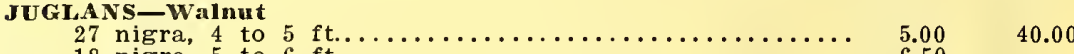

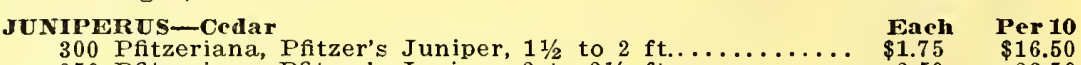

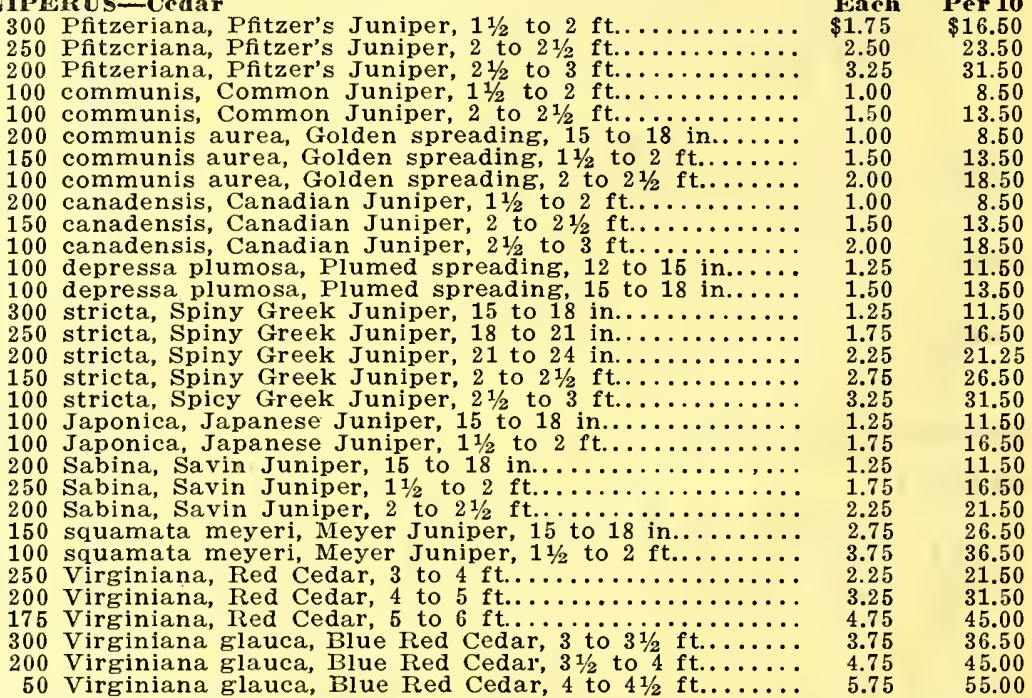

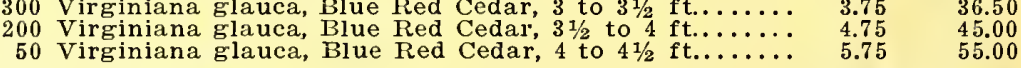

KErria-Corelhorus

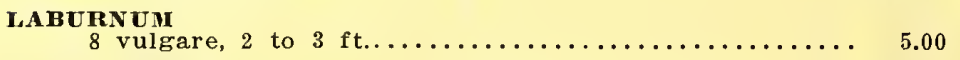

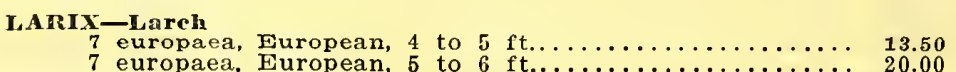

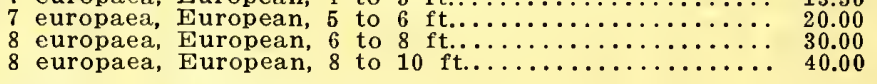

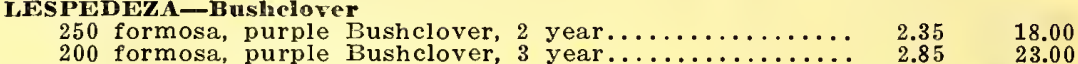

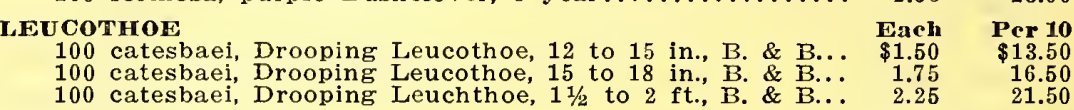

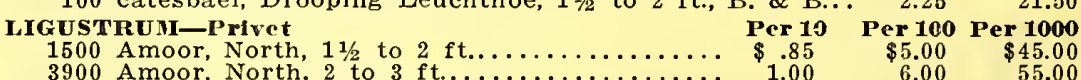

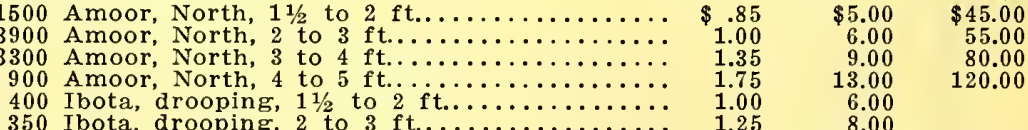

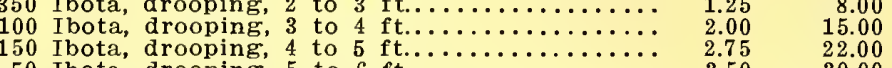

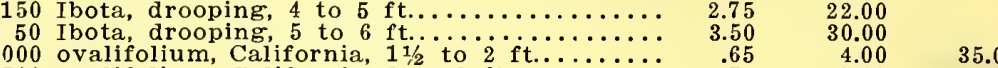

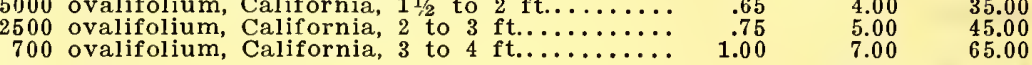

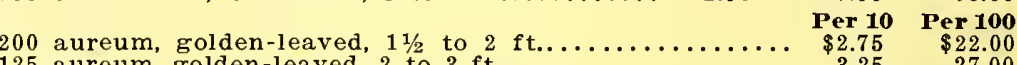

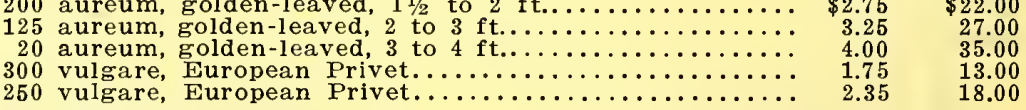

LIQUMDMMAAR-Sweet Gum

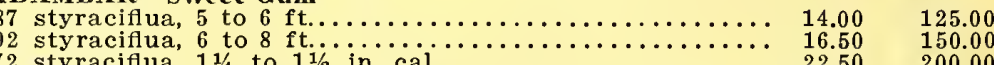

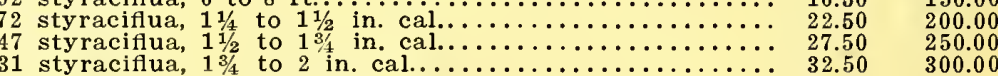

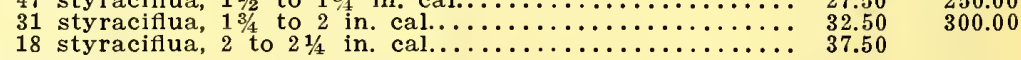

$\begin{array}{rr}7.00 & 60.00 \\ 8.50 & 75.00 \\ 0.00 & 90.00 \\ 1 & 150\end{array}$

299 tulipifera, 6 to 8 ft

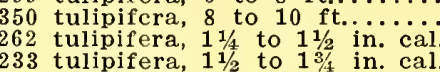

$\begin{array}{ll}2.25 & 17.00 \\ 2.75 & 22.00 \\ 1.50 & 10.00 \\ 2.00 & 14.00 \\ 1.75 & 12.00 \\ 2.15 & 16.00 \\ 2.75 & 22.00 \\ 1.50 & 10.00 \\ 2.00 & 14.00 \\ 2.35 & 18.00 \\ 1.50 & 10.00 \\ 2.00 & 14.00\end{array}$

albida, 2 to $3 \mathrm{ft}$

fragrantissima, white, 2 to $30 \mathrm{ft}$.

Morrowii, drooping, $1 / 2$ to 2 th

Ruprechtiana, red

Ruprechtiana, red-berried, 2 to 3

Ruprechtiana, red-berried, 4 to 4 to

tatarica alba, white, 2 to

150 tatarica alba, white, 4 to

215 tatarica rubra, red

415 tatarica rubra, red, 3 to

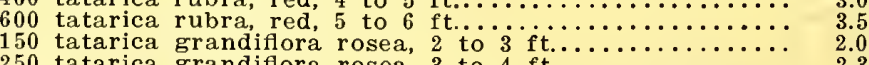

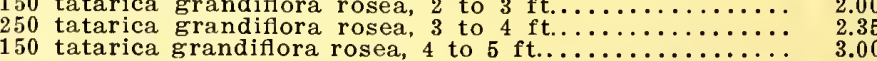

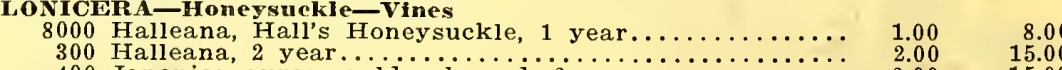

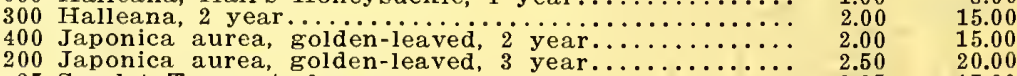

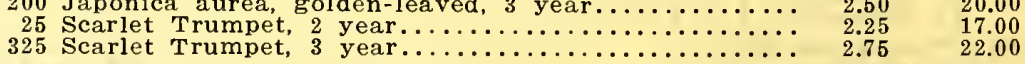

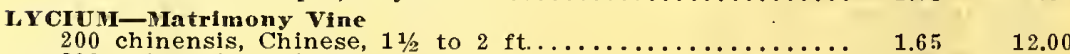

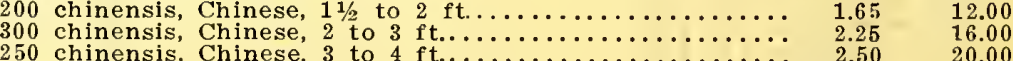


THOMAS B. MEEHAN CO., DRESHER, PA.-BULLETIN No, 1

MAGNOLIA

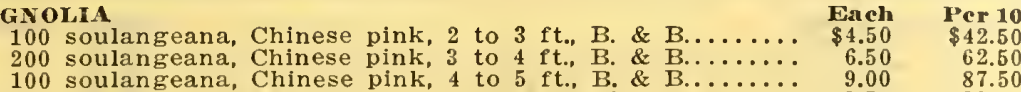

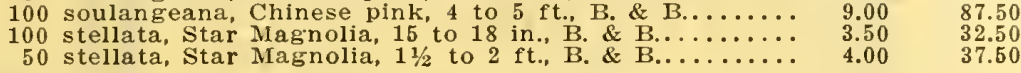
IAHONIA

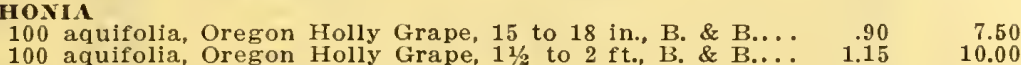

MALUS-(Pyrus)-Flowering Apple

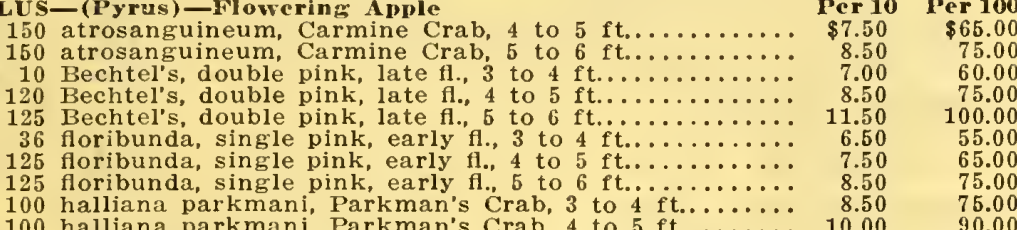

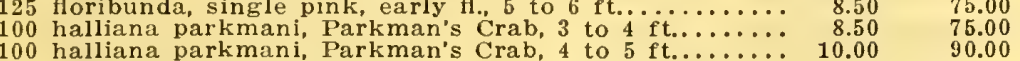

$\begin{array}{rl}9 & \text { nicdwitzkyana, single purple, purple-leaved, } 3 \text { to } 4 \text { ft. } \\ 16 & 6.50 \\ \text { niedwitzkyana, single purple, purple-1eaved, } 4 \text { to } 5 \mathrm{ftt} & 7.50\end{array}$

$\begin{array}{rlr}15 & \text { niedwitzkyana, single purple, purple-leaved, } 5 \text { to } 6 \mathrm{ft} \text {. } & 8.50 \\ 8 \text { nied witzkyana, single purple, purple-leaved, } 6 \text { to } 8 \mathrm{ft} . & 10.00 \\ 4 \text { scheideckeri, double pink, } 5 \text { to } 6 \text { it................. } & 8.50\end{array}$

MEEHA Y'S MALLOW MRVEL-Giant fl. Marsh Mallow

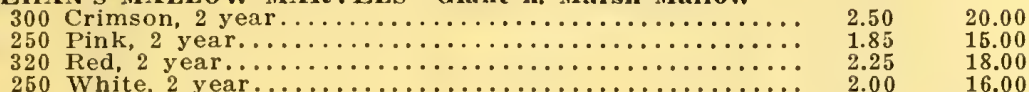

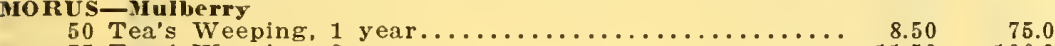

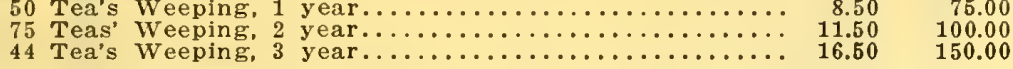

$\underset{200 \text { Carolinensis, }}{\text { MYRICA } \rightarrow \text { Rayberry }} 15$ to 18 in..................... $3.00 \quad 25.00$

NYSS

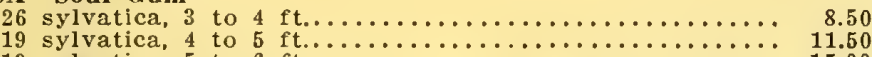

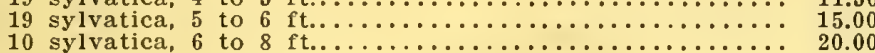

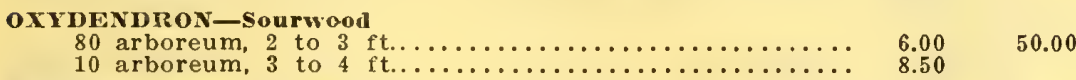

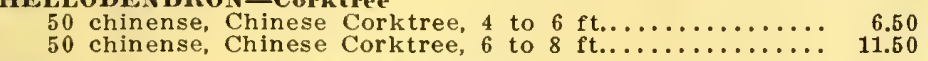

50.00
100.00

PHILADELPHUS-Syringa-Yock Orange

200 avalanche, Dwart, 2 to 3 ft..
150 avalanche, Dwarf, 3 to $4 \mathrm{ft}$.

600 coronarius, $11 / 2$ to 2
1575 coronarius,

150 aurea, Golden, 15 to 18 in.

50 grandiflorus, large flowered, $11 \% \ldots \ldots \ldots$

350 grandiflorus, large flowered, 2 to 3 ft.

00 Lemoinei, dwar

3 to 4

75 Mont Blanc,

225
speciosissimus,
speciosissimus,

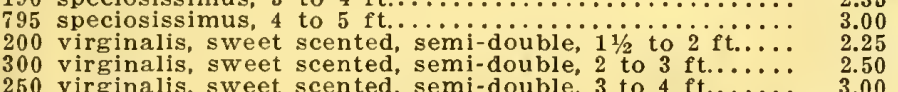
PHOTINIA

100 villosa, red-berried Photinia, 3 to 4 ft............... 5.00
75 villosa, red-berried Photinia, 4 to 5 ft..............

PICEA-Spruce

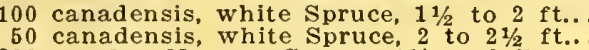

00 excelsa, Norway Spruce,

200 excelsa, Norway Spruce, 3 to $31 / 2$

75 pungens glauca, Colorado Blue, $11 / 2$ to

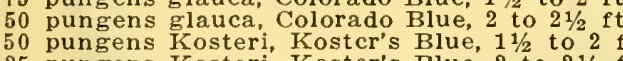

$1.50 \quad 100.00$

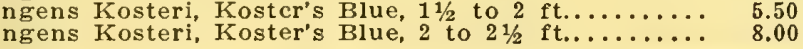

17.00
25.00
10.00
14.00
18.00
18.00
25.00
10.00
14.00
18.00
12.00
16.00
25.00
14.00
18.00
23.00
30.00
14.00
18.00
25.00
16.00
20.00

$\frac{40.00}{75.00}$

$\underset{\substack{\text { Per } 10.50 \\ 13.50}}{13.50}$

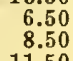

18.50
22.50
31.50

47.50
52.50
77.50

50 excelsa, Himalayan, 2 to $3 \mathrm{ft}$.
35 excelsa, Himalayan, 3 to $4 \mathrm{ft}$.
200 inala

200 Mughus, Mugho Pine, 12 to 15 .

Mughus, Mugho Pine, $11 / 2$ to $2 \mathrm{ft}$.

100 Mughus, Mugho Pine, 2 to $21 / 2$ ft. $\ldots \ldots$

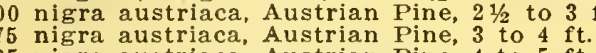

25 nigra austriaca, Austrian Pine, 4 to $5 \mathrm{ft}$

100 resinosa, Red Pine, 2 to 3

200 strobus, White Pine, 2 to 3

(1)

250 sylvestris, Scotch Pine, 2 to 3

ATANUS-Planes_-

2360 Orientalis, 6 to $8 \mathrm{ft}$....

677 Orentalis, $11 /$ to $11 / 2$ in. cal.
1122 Orientalis, $11 / 2$ to $13 / 4$ in. cal.

975 Orientalis, 13 to 2 in. cal..
278 Orientalis, 2 to $21 / 4$ in. cal..
14 Orientalis, $21 / 4$ to $21 \%$ in. cal.

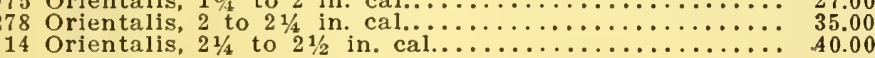

Populus-Popla

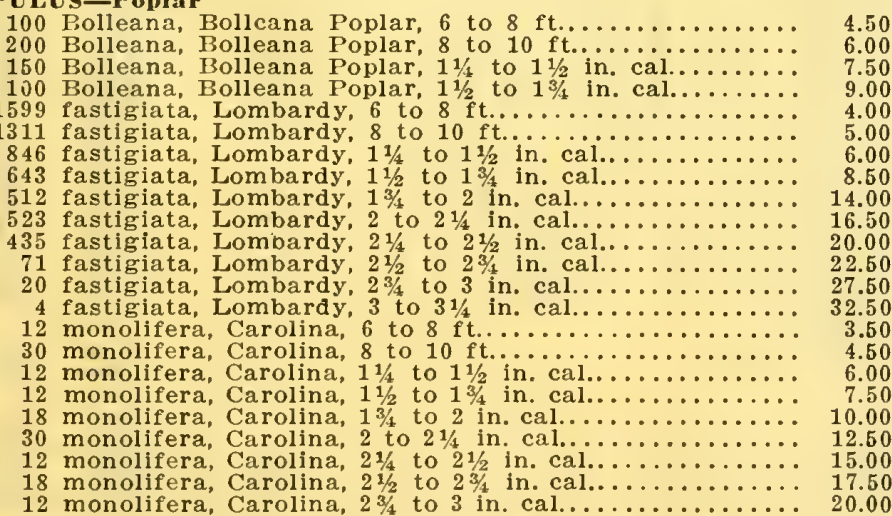

POTENTILLA-Shrubly Cincuefoil

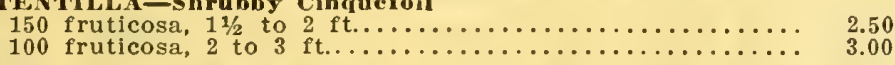

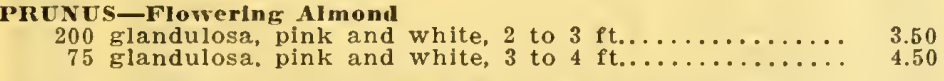

${ }_{25.00}^{20.00}$

30.00
40.00

PRUNUS-Flowering Plum

100 Pissardi, purple-leaved Plum, 4 to $5 \mathrm{ft}$.
100 Pissardi, purple-leaved Plum, 5 to $6 \mathrm{ft}$.

100 serrulata, Oriental Cherry, 4 to $5 \mathrm{ft}$

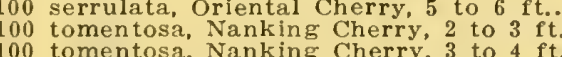

250 triloba, double pink, 2 to 3
150 triloba, double pink, 3 to

18.50
31.50
11.50
16.50
26.50
37.50
23.50
36.50
62.50
18.50
36.50
18.50
28.50
45.00
10.00
16.50
26.5

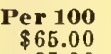

85.00
175.00
175.00

250.00
325.00

35.00
50.00
65.00
80.00
30.00
40.00
50.00
75.00
125.00
150.00
175.00
200.00

pYRACANTIIA-Fvergreen Fire Thorn-(See Crataegun)

$\begin{array}{ll} & \\ 5.00 & 40.00 \\ 6.50 & 50.00 \\ 66.00 & 50.00 \\ 8.50 & 75.00 \\ 3.00 & 25.00 \\ 4.00 & 35.00 \\ 4.00 & 30.00 \\ 5.00 & 40.00 \\ 50.50 & \end{array}$


7 alba, white, 6 to $8 \mathrm{ft}$
16 alba, white

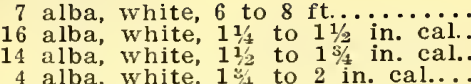

541 coccinea, scarlet, 6 to 8 ft.

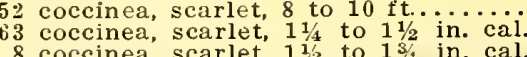

231 palustris, Pin, 6 to 8 ft

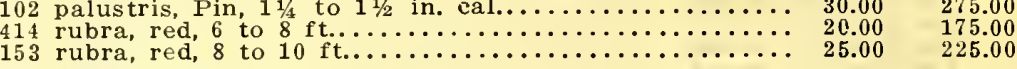

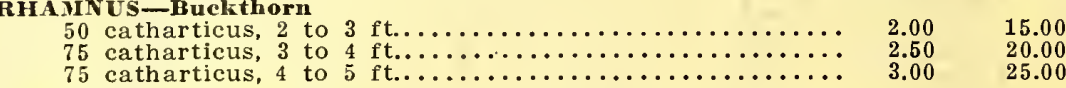

RHODOTYPOS - White IKerria $\quad \ldots \ldots \ldots \ldots \ldots \ldots \ldots \ldots, 200$

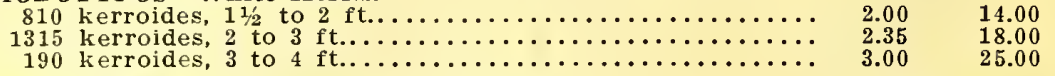

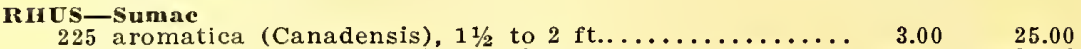

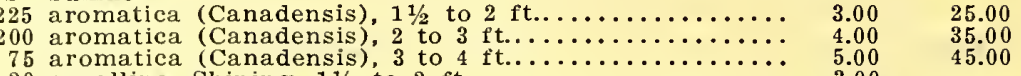

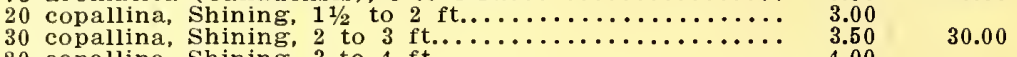

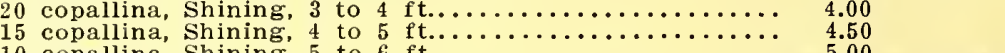

105 cotinus, 2 to 3 cotinus, 3 to

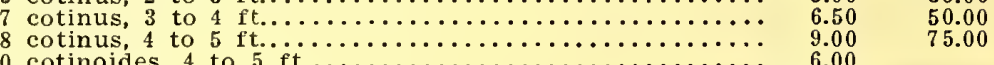

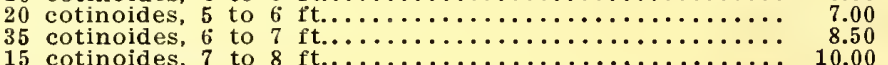

ROBINIA-Rosa acacia

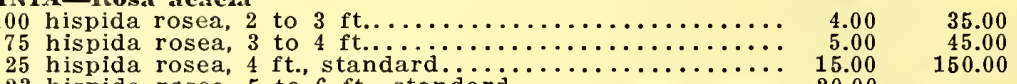

ROSA-Rose

250 blanda, Meadow Rose, $1 \frac{1 / 2}{\text { to }} 2$

200 lucida, Virginia Rose, $1 \frac{112}{20}$ to 200 lucida, Virginia Rose, 2 to 3

00 multiflora, Japanese Rose, 2 to 3 ft

75 nitida, Bristly Rose, $1 \frac{1 / 2}{2}$ to 2

150 palustris (Carolina), Sto

100 palustris (Carolina), Swamp Rose, 2 to $3 \mathrm{ft}$.

2500 rubiginosa, Sweetbrier, 2 to $3 \mathrm{ft}$.

150 rubrifolia, Redleaf, $11 / 2$ to 2 ft.

150 rubrifolia, Redleaf, 3 to 3 ft. $\mathrm{ft}$....

430 rugosa rubra, 2 to 3 ft...............................

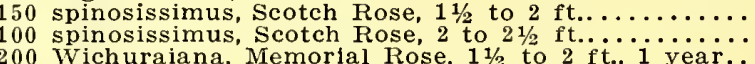

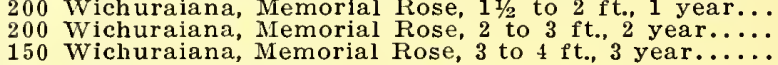

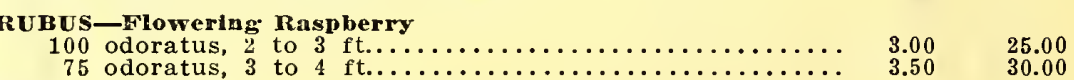

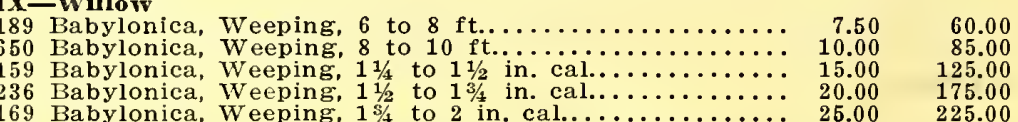

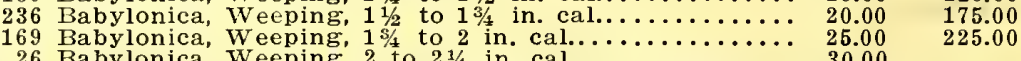

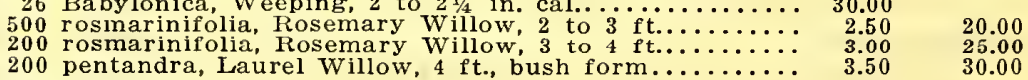

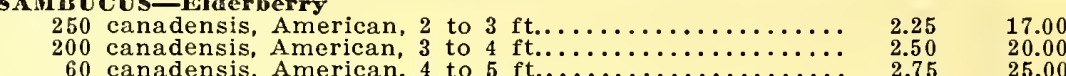

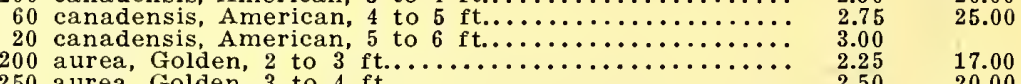

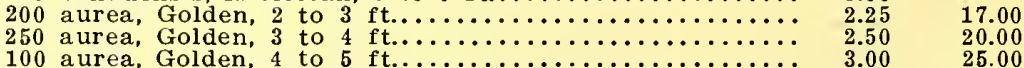

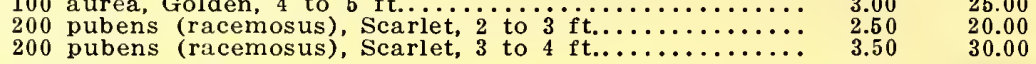

SORnARIA-False Spiraea

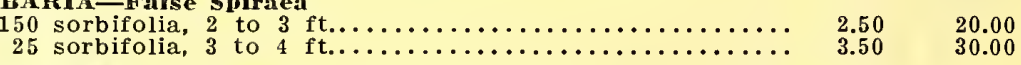

SORBUS-Nountain Ash

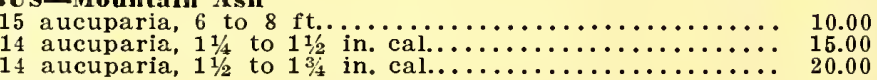

SPIRAEA

1000 Anthony Waterer, dwarf, pink, 10 to 12 in

115 Anthony Waterer, dwarf, pink, 15 to 18 i

650 arguta, $11 / 2$ to 2 ft.

50 arguta, 3 t

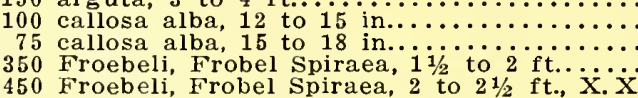

opulifolia, Ninebark, 2 to 3 ft.

opulifolia, Ninebark,

50 opulifolia aurea,

900 opulifolia aurea, 2 to 3 to 4 ft.
250 opulifolia aurea, 4 to $5 \mathrm{ft}$

90 prunifolia, Bridal Wreath, $11 \%$ to 2 ft

125 prunifolia, Bridal Wreath,

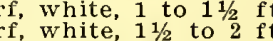

200 Thunbergii, dwarf, white, white, $21 / 2$ to $21 / 3 \mathrm{ft}$.

875 Van Houttei, white, white, 3 to 4 to 2 ft...........

3650 Van Houttei, white, 2 to 3 ft...............................

STEPHANANDR

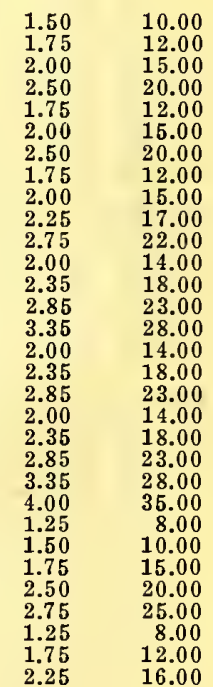

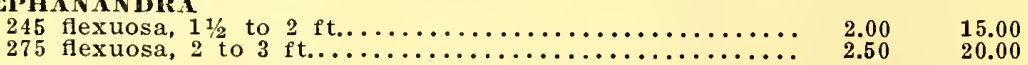

STY RAX

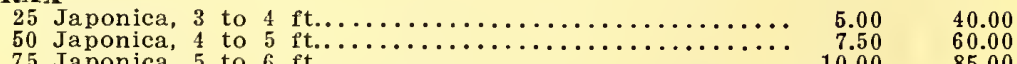

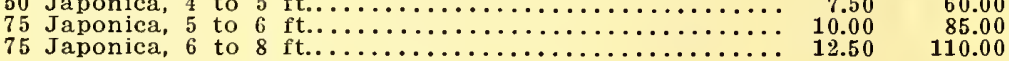

SYMPIORICARPOS-Snowberry-Coralberry

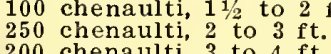

1125 racemosus, Snowberry, i $11 \ldots \ldots \ldots \ldots$

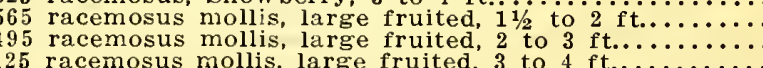

125 racemosus mollis, large fruited, 3 to $4 \mathrm{ft}$

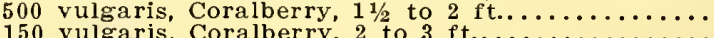

SYRING

$\begin{array}{ll}1.75 & 12.00 \\ 2.00 & 15.00 \\ 2.50 & 200 \\ 1.75 & 12.00 \\ 2.00 & 14.00 \\ 2.35 & 18.00 \\ 1.75 & 12.00 \\ 2.00 & 14.00 \\ 2.35 & 18.00 \\ 1.75 & 12.00\end{array}$

625 Rothomagensis, Persian purple, $1 \frac{1 / 2}{10} 2$

$\begin{array}{ll}2.00 & 15.00 \\ 2.35 & 18.00 \\ 2.85 & 23.00 \\ 2.50 & 20.00 \\ 3.50 & 30.00\end{array}$

150 saugeana, Purple Chinese, 2 to $3 \mathrm{ft}$.

250 Josikaea, Hungarian, 2 to 3 
THOMAS B. MEEHAN CO., DRESHER, PA.-BULLETIN No. 1

SYRINGA-Lilac-(Continued)

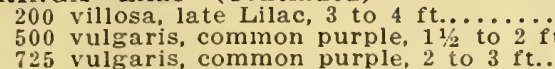

320 vulgaris, common purple, 3 to 4

100 vulgaris, common purple, 4 to $5 \mathrm{ft}$......
120 vulgaris, conmon purple, 5 to $6 \mathrm{ft} .$.
110 vulgaris alba, common whitc, 2 to 3

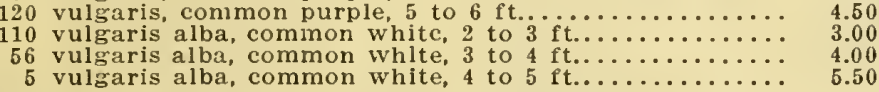

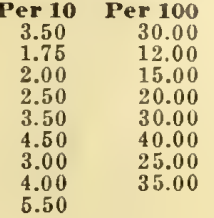

FRENCH LILACS-Named Varieties

These are all grown from cuttings and are on their own

roots. They will not sucker. The following list includes
many of the old favorites, as well as many of the best of the

Alphonsa Lavalle, double, light purple

Charles Joly, semi-double, red

Florent Stepman, single, white
Hugo Koster, single, pale lilac

Ludvio Spaeth, single reddish purpl

Marechal Lannes, semi-double, light bluish viole

Marie Le Gray, single, white

Mme. Casimier Perrier, double, white

Pres. Grevy, double, blue

William Robinson, doublc, violet pink

2 to 3 ft. whit

TAMARIX

$$
\begin{array}{rrrr}
2 & \text { to } & 3 & \mathrm{ft} \text { t. } \\
3 & \text { to } & 4 & \mathrm{ft} .
\end{array}
$$

50 odessana, 2 to 3
90 odessana, 3 to $4 \mathrm{f}$
100 odessana, 4 to $5 \mathrm{f}$.

TAXONIUM-Bald Cypress

rAXUS-Yew

100 repandens, Spreading English, 12 to 15 in

250 cuspidata, Spreading Japancse,

150 cuspidata, spreading Japanese, 2 to $21 / 2 \mathrm{ft}$............

200 nana (brevifolia), Divarp Japanese, 9 to 12 in...

IUXA-Arbor Vitae

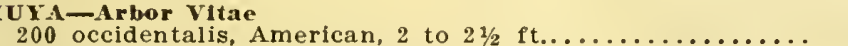

200 occidentalis, American, 2 to $21 \frac{1 / 2}{\mathrm{ft} .} .$.

400 occidentalis, American,

50 occidentalis, American,

occidentalis, American, 5 to 6 f

00 occidentalis, American, 6 to $7 \mathrm{ft}$........................

150 elegantissima, Golden-tipped, $21 / 2$ to $3 \mathrm{ft}$

250 globosa, Globe, 12 to 15 in

50 globosa, Globe, 15 to 18 in

hovosi, Hovey Arbor
hoveyi, Hovey Arbor

100
lutea, Geo. Peabody Arbor Vitae, $11 / 2$ to 2
155
lutea, Geo. Peabody Arbor Vitae. 2 to $21 / 2$

00 pyramidalis, Pyramidal, 2 to $21 / 2$

300 pyramidalis, Pyramidal, $21 / 2$ to 3

300 pyramidalis, Pyramida

50 pyramidalis, Pyramidal, 4 to $41 / 2$

rosenthali, Rosenthal Arbor Vitae, $1 \frac{1 / 2}{2}$ to 2

75 rosenthali, Rosenthal Aror Vita. 210 to $31 / 2$

W Wareana (Siberia)

Wareana (Siberia), Siberian, $21 / 2$ to $21 / 3$

0 Standishi. Standish Arbor Vitae, 2 to $21 / \frac{1}{\mathrm{f}}$

150 Standishi, Standish Arbor Vitae, $21 / 2$ to $3 \mathrm{ft}$

100 Orientalis, Oriental, 2 to $21 / 2$

200 Orientalis, Oriental, 3 to $31 / 2$

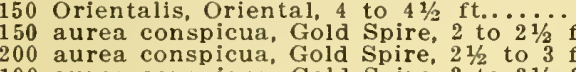

100 aurea conspicua, Gold Spire, 3 to $3 \frac{1 / 2}{2}$

300 aurea nana, Berckman's Golden, 12 to 15 in.
350 aurea nana, Berckman's Golden, 15 to 18 in...
250 aurea nana, Berckman's Golden, 18 to 21 in

250 elegantissima, Yellow Column, 2 to $2 \frac{1 / 2}{2}$

TILIA-Linden

200 Americana, American, 6 to $8 \mathrm{ft}$...
250 Americana, American, 8 to $10 \mathrm{ft}$.

200 Americana, American, $11 / \frac{1}{1}$ to $11 / 2$ in. cal...

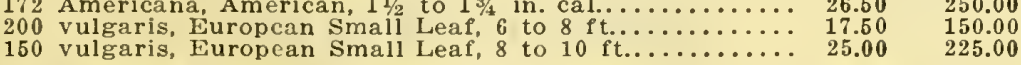

300 Canadensis, Canadian Hemlock, 2 to $21 / 2 \mathrm{Pt}$
250 Canadensls, Canadian Hemlock, $21 / 2$ to $3 \mathrm{f}$
150 Canadensis, Canadian Hemlock, 3 to $31 / 2 \mathrm{ft}$

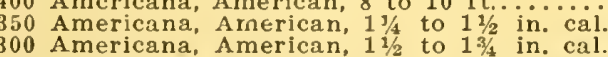

Americana, Amcrican,

Americana, American, 2 to $2 \frac{2}{2} \mathrm{ln}$. cal..

100 Moline, Moline Elm, 6 to $8 \mathrm{Pt}$

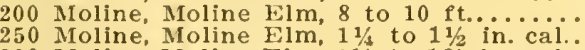

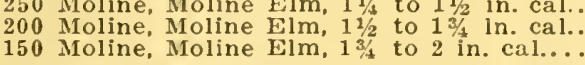

$\$ 6.00 \quad \$ 50.00$

$\$ .50$
85.00 $\quad 75.00$

$\$ 50.00$
75.00
100.00
150.00

250.00
325.00
425.00

75.00

100.00
125.00
200.00

100 corymbosum, Highbush Bluteberry, $1 \frac{1 / 2}{2}$ to $2 \mathrm{ft} \ldots \ldots \ldots$.......

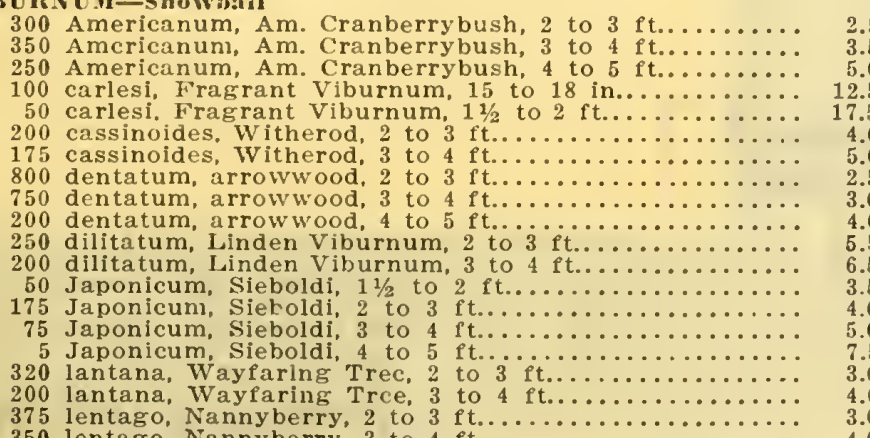


VIBURNUM-Snowball-(Continued)

410 lentago, Nannyberry, 4 to $5 \mathrm{ft}$.

150 lentago, Nannyberry, 5 to $6 \mathrm{ft}$.

150 nanum, Dwarf Cranberry, 6 to $9 \ldots \ldots \ldots \ldots \ldots \ldots \ldots$

100 nanum, Dwart Cranberry, 9 to 12 in.

800 opulus sterilis, common Snowball, $1 \frac{1 / 2}{2}$ to $2 . \ldots \ldots \ldots$

1400 opulus sterilis, common snowball, 2 to $3 \mathrm{ft}$

500 opulus sterilis, common snowball, 4 to $5 \mathrm{ft}$. 95 oxycoccos (opulus), High-bush Cranberry, 2 to $3 \mathrm{ft} .$. . 325 oxy coccos (opulus), High-bush Cranberry, 3 to $4 \mathrm{ft}$.. 325 oxycoccos (opulus), High-bush Cranberry, 4 to $5 \mathrm{ft}$..

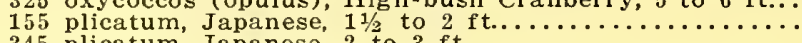

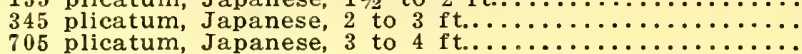

125 plicatum, Japanese, 4 to $5 \mathrm{ft} . \ldots \ldots \ldots \ldots \ldots \ldots \ldots \ldots \ldots \ldots \ldots$

100 rhytidophyllum, Leatherleaf Viburnum, 2 to $2 \frac{1}{2} \mathrm{ft}$. 75 rhytidiphyllum, Leatherleaf viburnum, $21 / 2$ to 3 ft., 25 rhytidophylium, Leatherleâ. viburnum, 3 to ${ }_{4} \ldots \ldots$

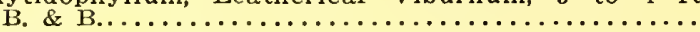

960 tomentosum, $1 \frac{1 / 2}{1}$ to $2 \mathrm{ft} . \ldots \ldots \ldots \ldots \ldots \ldots \ldots \ldots \ldots$

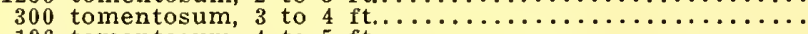

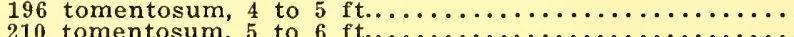

210 tomentosum

INCA-Periwinkle

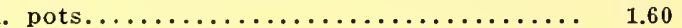

Per 10 Per 100

$5.50 \quad 45.00$

$\begin{array}{ll}6.50 & 55.00 \\ 2.50 & 20.00\end{array}$

3.50
3.85

$\begin{array}{ll}1.85 & 13.00 \\ 2.00 & 16.00\end{array}$

22.00
30.00

18.00

25.00
35.00

35.00
45.00

20.00

30.00
40.00

50.00

Per 10

$\$ 1.50 \$ 13.50$

$2.00 \quad 18.50$

$3.50 \quad 32.50$

Per 10 Per 100

$\$ 2.00 \$ 15.00$

3.00
4.00 $\quad \mathbf{3 5 . 0 0}$

5.00

$\mathbf{6 . 0 0} \quad \mathbf{5 5 . 0 0}$

VITEX_Chaste Tree

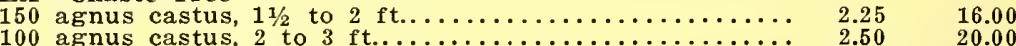

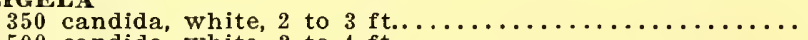

500 candida, white, 3 to 4 ft...

100 Desboisii, 2 to 3 ft. 5 .

100 Desboisii, 3 to $4 \mathrm{ft}$

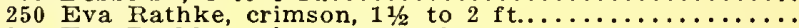

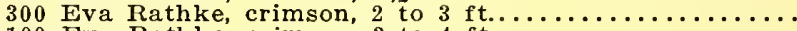

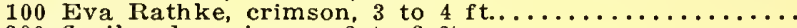

300 floribunda, crimson, 2 to $3 \mathrm{ft}$.

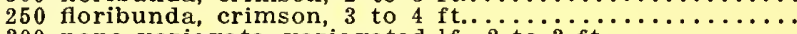

300 nana variegata, variegated lf., 2 to $3 \mathrm{ft} . \ldots \ldots \ldots \ldots \ldots$

250 nana variegata, variegated lf., 3 to $4 \mathrm{ft} . \ldots \ldots \ldots \ldots$

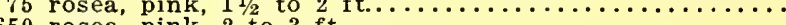

650 rosea, pink, 2 to 3 $\mathrm{ft}$.

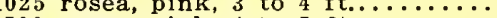

\begin{tabular}{l}
500 \\
125 \\
rosea, pink, 4 to $5 \mathrm{ft} . \ldots \ldots \ldots \ldots \ldots \ldots \ldots \ldots \ldots \ldots \ldots \ldots \ldots \ldots \ldots \ldots \ldots \ldots \ldots \ldots \ldots \ldots$ \\
\hline
\end{tabular}

11.00

15.00
20.00

20.00
30.00

30.00
15.00

20.00

25.00

15.00
20.00

30.00

20.00

25.00

15.00

20.00

12.00

15.00

20.00

25.00

30.00

220 sinensis, Chinese purple, 2 year............. $4.00 \quad 35.00$

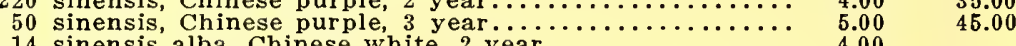

YUCCA-Adam's NeedIe

2400 filamentosa, 3 year.......................... 1.50

ZANTHORHIZA-Yellowwood

$1.50 \quad 12.00$

200 apiifolia, 9 to 12 in.

2.00

15.00

\section{GHINESE TONKIN CANES}

Nurserymen realize now that when they go to the labor and expense of planting a block of Shade Trees, it pays to have every tree produce revenue. Crooked and "bow-leg" trees are a dead loss. It pays to buy stakes and make every tree salable. First cost is very little as the stakes can be used over and over again.

We import our Cane Stakes direct from China.

Bale of 300 canes, $7 \mathrm{ft}$. long, $3 / 4$ to $7 / 8$ in. diameter at butt, $\$ 16.25$ per bale Bale of 300 canes, $10 \mathrm{ft}$. long, $3 / 4$ to $7 / 8$ in. diameter at butt, $\$ 24.50$ per bale

Sold in full bales only. Prices are for canes F. O. B. Dresher, Pa.

\section{DUTCH}

Finest material for pi loosen it up and add humus.

poses or for mixing with soil to $y$ is this necessary where ericaceous plants are propagated. Mixed with sand in your hot beds will give you a better stand of softwood cuttings and reduce fungus and damping off. For winter mulching of lawns and all kinds of flower and seed beds it is far superior to manure, as it does not have to be raked off in the spring and does not contain weed seeds.

We are handling only the very best quality. All brands are not alike in quality. Some brands appear to be cheaper, but there is a great difference in quantity packed in bale. Most cheap brands have 14 to 16 bushels per bale. Our bales run 20 bushels, dry weight. Therefore, in our bales you get a greater quantity of material.

$$
\begin{aligned}
& 1 \text { to } 9 \text { bales at } \$ 2.75 \text { per bale } \\
& 10 \text { bales and up at } \$ 2.60 \text { per bale } \\
& 25 \text { bales and up at } \$ 2.50 \text { per bale } \\
& \text { Special Quotations on Large Lots }
\end{aligned}
$$

All prices F. O. B. Dresher, $\mathrm{Pa}$. Prices subject to change without notice.

\section{RAFFIA}

We are HEADQUARTERS for Raffia for budding and tying purposes and our brands are the STANDARD of the Raffia market.

The LEADING nursery firms demand the best-A. A. Westcoastothers find X. X. X. Superior, X. X. Superior or Red Star suitable for their purpose.

Send for our price list before buying, or, better still, just place your order with us and we will give you our best price.

\section{NURSERY SUPPLIES}

We carry in stock a full line of Spades, Pruning and Budding Knives, Pruning Shears and Tree Callipers. Write for price list. 


\section{THOMAS B. MEEHAN CO.}

Wholesale Nurserymen

Sec. $4351 / 2$, P. L. $\&$ F.

DRESHER, MONTG. CO., PA.

Return Postage Guaranteed

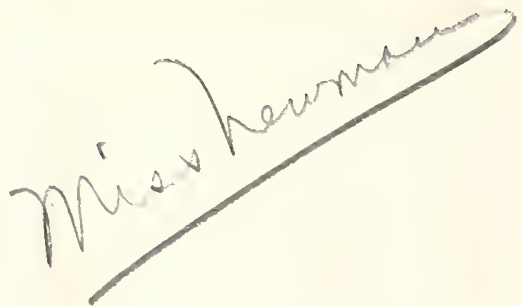

U. S. Department of Agriculture,

U. S. POSTAGE 1c Paid Dresher, Pa. Permit No. 1

\section{Washington, D. C.}

4. R. N.

Bureau of Plant Induatry Horticultural Crops and Diseases 